\title{
Extended supersymmetry with central charges in higher dimensional Dirac action
}

\author{
Yukihiro Fujimoto, ${ }^{1, *}$ Kouhei Hasegawa, ${ }^{2, \dagger}$ Kenji Nishiwaki, ${ }^{3,4, \$}$ Makoto Sakamoto, ${ }^{2, \S}$ \\ Kentaro Tatsumi, ${ }^{2, \|}$ and Inori Ueba ${ }^{2, \|}$ \\ ${ }^{1}$ National Institute of Technology, Oita College, Oita 870-0152, Japan \\ ${ }^{2}$ Department of Physics, Kobe University, Kobe 657-8501, Japan \\ ${ }^{3}$ Ruđer Bošković Institute, Division of Theoretical Physics, Zagreb 10000, Croatia \\ ${ }^{4}$ School of Physics, Korea Institute for Advanced Study, Seoul 02455, Republic of Korea
}

(Received 11 January 2019; published 11 March 2019)

\begin{abstract}
A new realization of extended quantum-mechanical supersymmetry (QM SUSY) with central extension is investigated. We first show that two different sets of $d+2(d+1)$ supercharges for $d=$ even (odd), each of which satisfies an $\mathcal{N}=d+2(d+1)$ extended QM SUSY algebra without central extension, are hidden in the four-dimensional mass spectrum of the $(4+d)$-dimensional Dirac action. We then find that the whole set of the supercharges forms an $\mathcal{N}=2 d+4(2 d+2)$ extended QM SUSY algebra with central charges for $d=$ even (odd). The representation of the supersymmetry algebra is shown to be 1/2-Bogomol'nyi-Prasad-Sommerfield states that correspond to a short representation for the supersymmetry algebra with a central extension. We explicitly examine the four-dimensional mass spectrum of the models with the hyperrectangle and the torus extra dimensions, and discuss their supersymmetric structures.
\end{abstract}

DOI: $10.1103 /$ PhysRevD.99.065002

\section{INTRODUCTION}

Quantum-mechanical supersymmetry (QM SUSY) is well known as the supersymmetry realized in quantum mechanics, which was introduced by Witten [1] to investigate the supersymmetry breaking. These days, the QM SUSY is applied to a wide range of research areas, e.g., exactly solvable systems in quantum mechanics [2-6], the Berry phase [7-9], black holes and AdS/CFT [10-14], the Sachdev-Ye-Kitaev model [15-19], extra dimensional models [20-25], and so on. Recent trends in QM SUSY are reviewed in Ref. [26].

One of the extensions of the QM SUSY is the $\mathcal{N}$-extended supersymmetry [27-34] and another one is the central extension of the supersymmetry algebra [35-38]. The $\mathcal{N}$-extended supersymmetry has $\mathcal{N}$ supercharges, each of which corresponds to a square root of

\footnotetext{
y-fujimoto@oita-ct.ac.jp

kouhei@phys.sci.kobe-u.ac.jp

knishiw@irb.hr

dragon@kobe-u.ac.jp

kentaro@stu.kobe-u.ac.jp

i-ueba@stu.kobe-u.ac.jp
}

Published by the American Physical Society under the terms of the Creative Commons Attribution 4.0 International license. Further distribution of this work must maintain attribution to the author(s) and the published article's title, journal citation, and DOI. Funded by SCOAP ${ }^{3}$. the Hamiltonian, and they lead to the degeneracy of the spectrum. In addition, the central extension of supersymmetry is an extension that introduces central charges into the supersymmetry algebra. ${ }^{1}$ Central charges are operators which commute with all the operators in the algebra, and they can make the size of supermultiplets small, compared with the regular representation $[43,44]$. Such multiplets are called short multiplets or Bogomol'nyiPrasad-Sommerfield (BPS) states, ${ }^{2}$ and especially, 1/2BPS states are constructed from half of the supercharges. Nevertheless, in quantum mechanics, not so many models which realize arbitrary large $\mathcal{N}$-extended QM SUSY with central charges are known. Thus, it is worthwhile to investigate a new realization of the $\mathcal{N}$-extended $\mathrm{QM}$ SUSY with central charges.

In Refs. [24,25], we have revealed that the $\mathcal{N}=2$ QM SUSY structure exists in the four-dimensional (4D) mass spectrum of the six-dimensional Dirac action. Since a higher dimensional Dirac spinor can be decomposed into 4D Dirac spinors with many "flavors" in the Kaluza-Klein (KK) decomposition, we expect that some symmetries larger than the $\mathcal{N}=2$ QM SUSY will be hidden in the 4D mass spectrum of higher dimensional Dirac actions.

\footnotetext{
${ }^{1}$ Spontaneous generations of the central charges in fieldtheoretic SUSY algebras and associated materials have been discussed (see, e.g., [39-42]).

${ }^{2}$ See also the original papers of BPS states $[45,46]$.
} 
Actually, it has been shown, in the previous paper [47], that the $\mathcal{N}=2$ QM SUSY can be extended to the $\mathcal{N}=2\lfloor d / 2\rfloor+2$ QM SUSY (but without central extension) for the $(4+d)$-dimensional Dirac action, where the symbol $\lfloor d / 2\rfloor$ denotes the largest integer less than or equal to $d / 2$.

In this paper, we discuss a new realization of $\mathcal{N}$-extended QM SUSY with central extension. Interestingly, we find another set of $2\lfloor d / 2\rfloor+2$ supercharges in the $(4+d)$-dimensional Dirac action, which forms the same $\mathcal{N}=2\lfloor d / 2\rfloor+2$ QM SUSY algebra as that given in Ref. [47]. Then, we show that the whole set of the $4\lfloor d / 2\rfloor+4$ supercharges forms an $\mathcal{N}=4\lfloor d / 2\rfloor+4$ extended QM SUSY algebra with central charges, and that the representation of the supersymmetry algebra forms a short multiplet corresponding to $1 / 2$-BPS states. We further verify that the supersymmetry clearly explains the structure of the 4D mass spectrum for the $(4+d)$ dimensional Dirac action in the hyperrectangle or the torus extra dimensions.

This paper is organized as follows: In Sec. II, we summarize the KK decomposition of a $(4+d)$-dimensional Dirac field and show that the $\mathcal{N}=2$ QM SUSY is hidden in the 4D mass spectrum. In Sec. III, we give two sets of $2\lfloor d / 2\rfloor+2$ supercharges, each of which satisfies the $2[d / 2\rfloor+2$ QM SUSY algebra without central charges, and then show that the whole set of $4\lfloor d / 2\rfloor+4$ supercharges forms the $\mathcal{N}=4\lfloor d / 2\rfloor+4$ QM SUSY algebra with central charges. In Sec. IV, we consider the representation of the algebra. Subsequently in Sec. V, we examine concrete examples which realize the $\mathcal{N}$-extended supersymmetry and confirm that the KK mode functions correspond to the representation given in Sec. IV. Section VI is devoted to summary and discussion.

\section{II. $\mathcal{N}=2$ QM SUSY IN HIGHER DIMENSIONAL DIRAC ACTION}

In this section, we give the setup of the $(4+d)$ dimensional Dirac action, and review the structure of the $\mathcal{N}=2$ QM SUSY hidden in the 4D mass spectrum of the system [47].

Let us consider the $(4+d)$-dimensional Dirac action ${ }^{3}$ with the 4D Minkowski space-time $M^{4}$ and a $d$-dimensional flat internal space $\Omega$ :

$$
\begin{aligned}
S= & \int_{M^{4}} d^{4} x \int_{\Omega} d^{d} y \bar{\Psi}(x, y)\left(i \Gamma^{\mu} \partial_{\mu}+i \Gamma^{y_{k}} \partial_{y_{k}}\right. \\
& -M \mathbf{1}_{\left.2^{[d / 2]+2}\right) \Psi(x, y),}
\end{aligned}
$$

where the coordinates of $M^{4}$ and $\Omega$ are represented by $x^{\mu}(\mu=0,1,2,3)$ and $y_{k}(k=1,2, \ldots, d)$, respectively.

\footnotetext{
${ }^{3}$ For earlier works on higher dimensional spinors, see, e.g., [48-50].
}

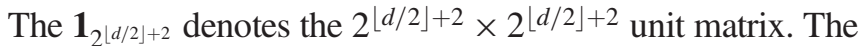
$\Gamma^{\mu}$ and $\Gamma^{y_{k}}$ indicate the $2^{\lfloor d / 2\rfloor+2} \times 2^{\lfloor d / 2\rfloor+2}$ gamma matrices in $(4+d)$-dimensions and satisfy the Clifford algebra

$$
\left\{\Gamma^{A}, \Gamma^{B}\right\}=-2 \eta^{A B} \mathbf{1}_{2^{\lfloor d / 2\rfloor+2}} \quad\left(A, B=0,1,2,3, y_{1}, \ldots, y_{d}\right),
$$

where $\eta^{A B}$ is the $(4+d)$-dimensional metric defined by $\eta_{A B}=\eta^{A B}=\operatorname{diag}(-1,+1, \ldots,+1)$. The parameter $M$ in the action (1) is a bulk mass and $\Psi(x, y)$ is a $(4+d)$ dimensional Dirac spinor with $2^{\lfloor d / 2\rfloor+2}$ components. The Dirac conjugate is defined as $\bar{\Psi}(x, y)=\Psi^{\dagger}(x, y) \Gamma^{0}$.

In this paper, we use the representation of the gamma matrices given by the direct product of the internal spin space and the 4D one, i.e.,

$\Gamma^{\mu}=\mathbf{1}_{2^{\lfloor d / 2\rfloor}} \otimes \gamma^{\mu}, \quad \Gamma^{y_{k}}=\gamma^{y_{k}} \otimes \gamma^{5} \quad(k=1,2, \ldots, d)$,

where $\gamma^{\mu}(\mu=0, \ldots, 3)$ denote the $4 \times 4$ 4D gamma matrices and $\gamma^{5} \equiv i \gamma^{0} \gamma^{1} \gamma^{2} \gamma^{3}$ denotes the 4D chiral matrix. The $\gamma^{y_{k}}(k=1,2, \ldots, d)$ are the $2^{\lfloor d / 2\rfloor} \times 2^{\lfloor d / 2\rfloor}$ internal

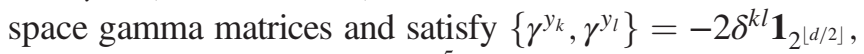
$\left(\gamma^{y_{k}}\right)^{\dagger}=-\gamma^{y_{k}}(k, l=1, \ldots, d){ }^{5}$

In terms of the $4 \mathrm{D}$ left-handed (right-handed) chiral spinors $\psi_{L, \alpha}^{(n)}(x)\left(\psi_{R, \alpha}^{(n)}(x)\right)$, the KK decomposition of the $(4+d)$-dimensional Dirac field $\Psi(x, y)$ will be given by

$\Psi(x, y)=\sum_{n} \sum_{\alpha}\left\{\boldsymbol{f}_{\alpha}^{(n)}(y) \otimes \psi_{L, \alpha}^{(n)}(x)+\boldsymbol{g}_{\alpha}^{(n)}(y) \otimes \psi_{R, \alpha}^{(n)}(x)\right\}$,

where the index $n$ indicates the $n$th level of the KK modes and $\alpha$ denotes the index that distinguishes the degeneracy of the $n$th KK modes (if it exists). The mode functions $\boldsymbol{f}_{\alpha}^{(n)}(y)\left(\boldsymbol{g}_{\alpha}^{(n)}(y)\right)$ have $2^{\lfloor d / 2\rfloor}$ components and are assumed to form a complete set with respect to the internal space associated with the 4D left-handed (right-handed) chiral spinors $\psi_{L, \alpha}^{(n)}(x)\left(\psi_{R, \alpha}^{(n)}(x)\right)$.

Substituting the expansion (4) into the action (1), we require that the action can be written into the form

$$
\begin{aligned}
S= & \int_{M^{4}} d^{4} x\left\{\sum_{\alpha} \sum_{n} \bar{\psi}_{\alpha}^{(n)}(x)\left(i \gamma^{\mu} \partial_{\mu}-m_{n}\right) \psi_{\alpha}^{(n)}(x)\right. \\
& +\sum_{\alpha} \bar{\psi}_{L, \alpha}^{(0)}(x) i \gamma^{\mu} \partial_{\mu} \psi_{L, \alpha}^{(0)}(x) \\
& \left.+\sum_{\alpha} \bar{\psi}_{R, \alpha}^{(0)}(x) i \gamma^{\mu} \partial_{\mu} \psi_{R, \alpha}^{(0)}(x)\right\},
\end{aligned}
$$

\footnotetext{
${ }^{4}$ We here adopt a slightly different representation of the gamma matrices from that given in the previous paper [47].

${ }^{5}$ For the case of $d=1$, we define $\gamma^{y_{1}}$ as $i$.
} 
where $\psi_{\alpha}^{(n)}(x)=\psi_{L, \alpha}^{(n)}(x)+\psi_{R, \alpha}^{(n)}(x)$ indicate 4D Dirac spinors with mass $m_{n}$ and $\psi_{L / R, \alpha}^{(0)}(x)$ are massless 4D chiral spinors. In order to obtain the above action, the mode functions $\boldsymbol{f}_{\alpha}^{(n)}(y)$ and $\boldsymbol{g}_{\alpha}^{(n)}(y)$ turn out to satisfy the following orthonormality relations:

$$
\begin{gathered}
\int_{\Omega} d^{d} y \boldsymbol{f}_{\alpha}^{(n) \dagger}(y) \boldsymbol{f}_{\beta}^{(m)}(y)=\int_{\Omega} d^{d} y \boldsymbol{g}_{\alpha}^{(n) \dagger}(y) \boldsymbol{g}_{\beta}^{(m)}(y)=\delta_{\alpha \beta} \delta^{n m}, \\
\int_{\Omega} d^{d} y \boldsymbol{f}_{\alpha}^{(n) \dagger}(y)\left(A \boldsymbol{g}_{\beta}^{(m)}(y)\right)=\int_{\Omega} d^{d} y \boldsymbol{g}_{\alpha}^{(n) \dagger}(y)\left(A^{\dagger} \boldsymbol{f}_{\beta}^{(m)}(y)\right)=m_{n} \delta_{\alpha \beta} \delta^{n m},
\end{gathered}
$$

where $A=-i \gamma^{y_{k}} \partial_{y_{k}}+M \mathbf{1}_{2^{\lfloor d / 2\rfloor}}$ and $A^{\dagger}=i \gamma^{y_{k}} \partial_{y_{k}}+M \mathbf{1}_{2^{\lfloor d / 2\rfloor}}{ }^{6}$. Since the mode functions $\boldsymbol{f}_{\alpha}^{(n)}(y)$ and $\boldsymbol{g}_{\alpha}^{(n)}(y)$ are assumed to form the complete sets, the relations (6) lead to

$$
\begin{aligned}
& Q\left(\begin{array}{c}
\boldsymbol{f}_{\alpha}^{(n)}(y) \\
0
\end{array}\right)=m_{n}\left(\begin{array}{c}
0 \\
\boldsymbol{g}_{\alpha}^{(n)}(y)
\end{array}\right), \quad H\left(\begin{array}{c}
\boldsymbol{f}_{\alpha}^{(n)}(y) \\
0
\end{array}\right)=m_{n}^{2}\left(\begin{array}{c}
\boldsymbol{f}_{\alpha}^{(n)}(y) \\
0
\end{array}\right), \quad(-1)^{F}\left(\begin{array}{c}
\boldsymbol{f}_{\alpha}^{(n)}(y) \\
0
\end{array}\right)=-\left(\begin{array}{c}
\boldsymbol{f}_{\alpha}^{(n)}(y) \\
0
\end{array}\right) \\
& Q\left(\begin{array}{c}
0 \\
\boldsymbol{g}_{\alpha}^{(n)}(y)
\end{array}\right)=m_{n}\left(\begin{array}{c}
\boldsymbol{f}_{\alpha}^{(n)}(y) \\
0
\end{array}\right), \quad H\left(\begin{array}{c}
0 \\
\boldsymbol{g}_{\alpha}^{(n)}(y)
\end{array}\right)=m_{n}^{2}\left(\begin{array}{c}
0 \\
\boldsymbol{g}_{\alpha}^{(n)}(y)
\end{array}\right), \quad(-1)^{F}\left(\begin{array}{c}
0 \\
\boldsymbol{g}_{\alpha}^{(n)}(y)
\end{array}\right)=+\left(\begin{array}{c}
0 \\
\boldsymbol{g}_{\alpha}^{(n)}(y)
\end{array}\right),
\end{aligned}
$$

where the supercharge $Q$, the Hamiltonian $H$, and the "fermion" number operator $(-1)^{F}$ are defined as

$$
Q=\left(\begin{array}{cc}
0 & A \\
A^{\dagger} & 0
\end{array}\right), \quad H=Q^{2}=\left(\begin{array}{cc}
\left(-\partial_{y}^{2}+M^{2}\right) \mathbf{1}_{2^{\lfloor d / 2\rfloor}} & 0 \\
0 & \left(-\partial_{y}^{2}+M^{2}\right) \mathbf{1}_{2^{\lfloor d / 2\rfloor}}
\end{array}\right), \quad(-1)^{F}=\left(\begin{array}{cc}
-\mathbf{1}_{2^{\lfloor d / 2\rfloor}} & 0 \\
0 & \mathbf{1}_{2^{\lfloor d / 2\rfloor}}
\end{array}\right) .
$$

The Eqs. (7) are nothing but the relations of $\mathcal{N}=2$ supersymmetric quantum mechanics ${ }^{7}[1,2]$, and the mode functions $\left(\boldsymbol{f}_{\alpha}^{(n)}(y), 0\right)^{\mathrm{T}}$ and $\left(0, \boldsymbol{g}_{\alpha}^{(n)}(y)\right)^{\mathrm{T}}$ correspond to the "bosonic" and "fermionic" states that form an $\mathcal{N}=2$ supermultiplet in the supersymmetric quantum mechanics. Thus, we have found that the $\mathcal{N}=2$ QM SUSY is hidden in the KK mode functions and the 4D mass spectrum.

We should notice that the supercharge $Q$ has to be Hermitian to realize the $\mathcal{N}=2$ QM SUSY. The Hermitian property of the supercharge $Q$ is assured if the KK mode functions satisfy the condition for the surface integral

$$
\int_{\partial \Omega} d^{d-1} y \boldsymbol{f}_{\alpha}^{(n) \dagger}(y) i n_{y_{k}}(y) \gamma^{y_{k}} \boldsymbol{g}_{\beta}^{(m)}(y)=0
$$

for all $m, n, \alpha, \beta$. The $n_{y_{k}}(y)$ is a normal unit vector on the boundary $\partial \Omega$. Since the above equation can be derived from the action principle $\delta S=0$, the Hermiticity of the supercharge $Q$ is guaranteed as long as the Dirac field obeys the action principle. Thus, the $\mathcal{N}=2$ QM SUSY is always realized in the $4 \mathrm{D}$ mass spectrum of the higher

\footnotetext{
${ }^{6}$ It is noted that the definition of $A$ (and $A^{\dagger}$ ) is different from that in [47] as

$$
-\Gamma^{0}\left(i \Gamma^{y_{k}} \partial_{y_{k}}-M \mathbf{1}_{2^{\lfloor d / 2\rfloor+2}}\right) \equiv\left(\begin{array}{cc}
0 & A \\
A^{\dagger} & 0
\end{array}\right) \otimes \mathbf{1}_{2} .
$$

${ }^{7}$ If one defines $Q_{1}=Q$ and $Q_{2}=i(-1)^{F} Q$, then they form the $\mathcal{N}=2$ SUSY algebra $\left\{Q_{j}, Q_{k}\right\}=2 H \delta_{j k}$ for $j, k=1,2$.
}

dimensional Dirac action and the doubly degenerate states $\left(\boldsymbol{f}_{\alpha}^{(n)}(y), 0\right)^{\mathrm{T}}$ and $\left(0, \boldsymbol{g}_{\alpha}^{(n)}(y)\right)^{\mathrm{T}}$ are mutually related by the supercharge $Q$, except for zero energy states.

\section{III. $\mathcal{N}$-EXTENDED SUPERSYMMETRY WITH CENTRAL CHARGES}

Although we have succeeded in explaining the degeneracy between the mode functions $\boldsymbol{f}_{\alpha}^{(n)}(y)$ and $\boldsymbol{g}_{\alpha}^{(n)}(y)$ from an $\mathcal{N}=2$ supersymmetry point of view, we will see further degeneracy labeled by $\alpha$ in the 4D mass spectrum. We then expect that some structures will be hidden furthermore in the 4D mass spectrum. Actually, in the previous paper [47], we have revealed that an $\mathcal{N}=2\lfloor d / 2\rfloor+2$ extended QM SUSY is hidden in the 4D mass spectrum.

In this section, we first point out that there exist two sets of the supercharges, each of which forms the $\mathcal{N}=$ $2\lfloor d / 2\rfloor+2$ extended QM SUSY algebra without central extension. We then show that the whole set of the supercharges satisfies the $\mathcal{N}=4\lfloor d / 2\rfloor+4$ QM SUSY algebra with central charges. In the next subsection, we clarify the representation of the algebra.

\section{A. $\mathcal{N}=2\lfloor d / 2\rfloor+2$ supersymmetry for algebraic and geometric extensions}

In this subsection, we explicitly construct two sets of supercharges, where one is called algebraic and the other is geometric, respectively. We then show that each set of them satisfies the $\mathcal{N}=2\lfloor d / 2\rfloor+2$ supersymmetry algebra without central charges. 
(i) Algebraic supercharges

The $\mathcal{N}=2\lfloor d / 2\rfloor+2$ supercharges based on the algebraic property of the gamma matrices are defined as follows:

$$
\begin{aligned}
Q_{k}= & \left(\begin{array}{cc}
0 & -i \gamma^{d+1} \gamma^{y_{k}} A \\
i A^{\dagger} \gamma^{d+1} \gamma^{y_{k}} & 0
\end{array}\right), \quad Q_{d+1}=Q, \quad Q_{d+2}=\left(\begin{array}{cc}
0 & -i \gamma^{d+1} A \\
i A^{\dagger} \gamma^{d+1} & 0
\end{array}\right), \\
& (k=1,2, \ldots, d),
\end{aligned}
$$

for $d=$ even, and

$$
\begin{aligned}
Q_{k}= & \left(\begin{array}{cc}
0 & \gamma^{y_{d}} \gamma^{y_{k}} A \\
-A^{\dagger} \gamma^{y_{d}} \gamma^{y_{k}} & 0
\end{array}\right), \quad Q_{d}=Q, \quad Q_{d+1}=\left(\begin{array}{cc}
0 & \gamma^{y_{d}} A \\
-A^{\dagger} \gamma^{y_{d}} & 0
\end{array}\right), \\
& (k=1,2, \ldots, d-1),
\end{aligned}
$$

for $d=$ odd. It should be noticed that $\gamma^{d+1} \equiv i^{d / 2} \gamma^{y_{1}} \cdots \gamma^{y_{d}}$ can be introduced only for $d=$ even and corresponds to the internal chiral matrix which satisfies $\left\{\gamma^{d+1}, \gamma^{y_{k}}\right\}=0,\left(\gamma^{d+1}\right)^{2}=\mathbf{1}_{2^{\lfloor d / 2\rfloor}}$ and $\left(\gamma^{d+1}\right)^{\dagger}=\gamma^{d+1}$. On the other hand, in the odd $d$ dimensions, one of $\gamma^{y_{k}}(k=1,2, \ldots, d)$ should be represented by the product of all the other gamma matrices. For the following sections, we use the representation of $\gamma^{y_{d}}=-i^{(d+1) / 2} \gamma^{y_{1}} \cdots \gamma^{y_{d-1}}$ for $d=$ odd with

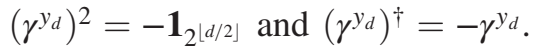

The above supercharges are found to satisfy the $\mathcal{N}=2\lfloor d / 2\rfloor+2$ supersymmetry algebra without central extension, i.e.,

$$
\left\{Q_{i}, Q_{j}\right\}=2 H \delta_{i j}, \quad\left[H, Q_{i}\right]=0 \quad(i, j=1,2, \ldots, 2\lfloor d / 2\rfloor+2) .
$$

(ii) Geometric supercharges

Another set of the $\mathcal{N}=2\lfloor d / 2\rfloor+2$ supercharges can be constructed, by use of the internal gamma matrices together with the reflection operators of the internal space $\Omega$, as follows:

$$
\begin{aligned}
\tilde{Q}_{k}= & \left(\begin{array}{cc}
0 & -i \gamma^{d+1} \gamma^{y_{k}} R_{k} A \\
i A^{\dagger} \gamma^{d+1} \gamma^{y_{k}} R_{k} & 0
\end{array}\right), \quad \tilde{Q}_{d+1}=\left(\begin{array}{cc}
0 & P A \\
A^{\dagger} P & 0
\end{array}\right), \quad \tilde{Q}_{d+2}=\left(\begin{array}{cc}
0 & -i \gamma^{d+1} P A \\
i A^{\dagger} \gamma^{d+1} P & 0
\end{array}\right), \\
& (k=1,2, \ldots, d),
\end{aligned}
$$

for $d=$ even, and

$$
\begin{aligned}
\tilde{Q}_{k}= & \left(\begin{array}{cc}
0 & \gamma^{y_{d}} \gamma^{y_{k}} R_{d} R_{k} A \\
-A^{\dagger} \gamma^{y_{d}} \gamma^{y_{k}} R_{d} R_{k} & 0
\end{array}\right), \quad \tilde{Q}_{d}=\left(\begin{array}{cc}
0 & P A \\
A^{\dagger} P & 0
\end{array}\right), \quad \tilde{Q}_{d+1}=\left(\begin{array}{cc}
0 & \gamma^{y_{d}} R_{d} P A \\
-A^{\dagger} \gamma^{y_{d}} R_{d} P & 0
\end{array}\right), \\
& (k=1,2, \ldots, d-1),
\end{aligned}
$$

for $d=$ odd. The $R_{k}(k=1,2, \ldots, d)$ represents the reflection operator for the $y_{k}$ direction, ${ }^{8}$ and $P=\prod_{k=1}^{d} R_{k}$ denotes the point reflection (or parity) operator of the internal space. The above supercharges also realize the $\mathcal{N}=2\lfloor d / 2\rfloor+2$ supersymmetry algebra without central extension

$$
\left\{\tilde{Q}_{i}, \tilde{Q}_{j}\right\}=2 H \delta_{i j}, \quad\left[H, \tilde{Q}_{i}\right]=0 \quad(i, j=1,2, \ldots, 2\lfloor d / 2\rfloor+2) .
$$

It should be noted that the supercharges $\tilde{Q}_{i}$ are the same as those obtained in the previous paper [47], except for $\tilde{Q}_{d+1}$ for $d=$ even and $\tilde{Q}_{d}$ for $d=$ odd.

\footnotetext{
${ }^{8}$ The reflection operator $R_{k}(k=1,2, \ldots, d)$ is defined by $\left(R_{k} f\right)\left(y_{1}, \ldots, y_{k}, \ldots, y_{d}\right) \equiv f\left(y_{1}, \ldots,-y_{k}, \ldots, y_{d}\right)$ for any function $f\left(y_{1}, \ldots, y_{d}\right)$. The $R_{k}$ and $\partial_{l}$ satisfy $R_{k} \partial_{l}=\left(1-2 \delta_{k l}\right) \partial_{l} R_{k}$.
} 


\section{B. $\mathcal{N}=4\lfloor d / 2\rfloor+4$ supersymmetry with central charges}

In the previous subsection, we have seen that both of the supercharges $Q_{i}$ and $\tilde{Q}_{i}(i=1,2, \ldots, 2\lfloor d / 2\rfloor+2)$ satisfy the same $\mathcal{N}=2\lfloor d / 2\rfloor+2$ QM SUSY algebra without the central extension. Here, we show that the supercharges $Q_{i}$ together with $\tilde{Q}_{i}(i=1,2, \ldots, 2\lfloor d / 2\rfloor+2)$ can extend the algebra to the $\mathcal{N}=4\lfloor d / 2\rfloor+4$ QM SUSY algebra with central charges $\left(Z_{i}\right)$ such that

$$
\begin{aligned}
\left\{Q_{i}, Q_{j}\right\} & =\left\{\tilde{Q}_{i}, \tilde{Q}_{j}\right\}=2 H \delta_{i j}, \\
\left\{Q_{i}, \tilde{Q}_{j}\right\} & =2 Z_{i} \delta_{i j}, \\
{\left[Z_{i}, Q_{j}\right] } & =\left[Z_{i}, \tilde{Q}_{j}\right]=\left[Z_{i}, Z_{j}\right]=\left[Z_{i}, H\right]=\left[H, Q_{i}\right] \\
& =\left[H, \tilde{Q}_{i}\right]=0, \\
(i, j & =1,2, \ldots, 2\lfloor d / 2\rfloor+2),
\end{aligned}
$$

where $Z_{i}(i=1,2, \ldots, 2\lfloor d / 2\rfloor+2)$ are given by

$$
\begin{gathered}
Z_{k}=Q_{k} \tilde{Q}_{k}=\left(\begin{array}{cc}
A A^{\dagger} R_{k} & 0 \\
0 & A^{\dagger} R_{k} A
\end{array}\right) \quad(k=1,2, \ldots, d), \\
Z_{d+1}=Q_{d+1} \tilde{Q}_{d+1}=\left(\begin{array}{cc}
A A^{\dagger} P & 0 \\
0 & A^{\dagger} P A
\end{array}\right), \\
Z_{d+2}=Q_{d+2} \tilde{Q}_{d+2}=\left(\begin{array}{cc}
A A^{\dagger} P & 0 \\
0 & A^{\dagger} P A
\end{array}\right),
\end{gathered}
$$

for $d=$ even, and

$$
\begin{aligned}
Z_{k}= & Q_{k} \tilde{Q}_{k}=\left(\begin{array}{cc}
A A^{\dagger} R_{d} R_{k} & 0 \\
0 & A^{\dagger} R_{d} R_{k} A
\end{array}\right) \\
& (k=1,2, \ldots, d-1), \\
Z_{d}= & Q_{d} \tilde{Q}_{d}=\left(\begin{array}{cc}
A A^{\dagger} P & 0 \\
0 & A^{\dagger} P A
\end{array}\right), \\
Z_{d+1}= & Q_{d+1} \tilde{Q}_{d+1}=\left(\begin{array}{cc}
A A^{\dagger} R_{d} P & 0 \\
0 & A^{\dagger} R_{d} P A
\end{array}\right),
\end{aligned}
$$

for $d=$ odd. ${ }^{9}$ Since $Z_{i}(i=1,2, \ldots, 2\lfloor d / 2\rfloor+2)$ commute with all the operators, we can regard $Z_{i}$ as central charges in this algebra. As we can see the form of the central charges, they may be interpreted as the reflection operators (accompanied with the Hamiltonian) compatible with the QM SUSY.

In the next section, we discuss the representation of the supersymmetry algebra. For this purpose, it is convenient to adopt the basis of supercharges as

$$
Q_{i}^{ \pm}=\frac{1}{2}\left(Q_{i} \pm \tilde{Q}_{i}\right) \quad(i=1,2, \ldots, 2\lfloor d / 2\rfloor+2) .
$$

The explicit forms of the supercharges $Q_{i}^{ \pm}$are given as

$$
\begin{aligned}
Q_{k}^{ \pm}= & \left(\begin{array}{cc}
0 & -i \gamma^{d+1} \gamma^{y_{k}} \Pi_{k}^{ \pm} A \\
i A^{\dagger} \gamma^{d+1} \gamma^{y_{k}} \Pi_{k}^{ \pm} & 0
\end{array}\right), \quad Q_{d+1}^{ \pm}=\left(\begin{array}{cc}
0 & \Pi_{d+1}^{ \pm} A \\
A^{\dagger} \Pi_{d+1}^{ \pm} & 0
\end{array}\right), \quad Q_{d+2}^{ \pm}=\left(\begin{array}{cc}
0 & -i \gamma^{d+1} \Pi_{d+1}^{ \pm} A \\
i A^{\dagger} \gamma^{d+1} \Pi_{d+1}^{ \pm} & 0
\end{array}\right), \\
& (k=1,2, \ldots, d),
\end{aligned}
$$

for $d=$ even, and

$$
\begin{aligned}
Q_{k}^{ \pm}= & \left(\begin{array}{cc}
0 & \gamma^{y_{d}} \gamma^{y_{k}} \Pi_{d k}^{ \pm} A \\
-A^{\dagger} \gamma^{y_{d}} \gamma^{y_{k}} \Pi_{d k}^{ \pm} & 0
\end{array}\right), \quad Q_{d}^{ \pm}=\left(\begin{array}{cc}
0 & \Pi_{d+1}^{ \pm} A \\
A^{\dagger} \Pi_{d+1}^{ \pm} & 0
\end{array}\right), \quad Q_{d+1}^{ \pm}=\left(\begin{array}{cc}
0 & \gamma^{y_{d}} \Pi_{d(d+1)}^{ \pm} A \\
-A^{\dagger} \gamma^{y_{d}} \Pi_{d(d+1)}^{ \pm} & 0
\end{array}\right), \\
& (k=1,2, \ldots, d-1),
\end{aligned}
$$

for $d=$ odd, where $\Pi_{k}^{ \pm}=\left(1 \pm R_{k}\right) / 2, \Pi_{d+1}^{ \pm}=(1 \pm P) / 2$, $\Pi_{d k}^{ \pm}=\left(1 \pm R_{d} R_{k}\right) / 2$, and $\Pi_{d(d+1)}^{ \pm}=\left(1 \pm R_{d} P\right) / 2$ play the role of the projection operators. Then, these supercharges are found to satisfy the algebra

$$
\begin{gathered}
\left\{Q_{i}^{ \pm}, Q_{j}^{ \pm}\right\}=\left(H \pm Z_{i}\right) \delta_{i j}, \\
\left\{Q_{i}^{ \pm}, Q_{j}^{\mp}\right\}=0,(i, j=1,2, \ldots, 2\lfloor d / 2\rfloor+2) .
\end{gathered}
$$

\footnotetext{
${ }^{9}$ The supercharges especially satisfy $Q_{i} \tilde{Q}_{i}=\tilde{Q}_{i} Q_{i}(i=1$, $2, \ldots, 2\lfloor d / 2\rfloor+2)$.
}

In the next section, we use this basis of the supercharges. It should be pointed out that all of the supercharges $Q_{i}$ and $\tilde{Q}_{i}\left(\right.$ or $\left.Q_{i}^{ \pm}\right)(i=1,2, \ldots, 2\lfloor d / 2\rfloor+2)$ would not be necessarily well defined in the system. In order for them to be well defined, the supercharges $Q_{i}$ and $\tilde{Q}_{i}$ have to be compatible with boundary conditions (if the internal space $\Omega$ has boundaries), that is, for any state $\Phi(y), Q_{i} \Phi(y)$ and $\tilde{Q}_{i} \Phi(y)$ should obey the same boundary condition as that on $\Phi(y)$, otherwise the action of $Q_{i}$ and $\tilde{Q}_{i}$ on $\Phi(y)$ is ill defined. Furthermore, in order for $\tilde{Q}_{i}$ to be well defined, the reflection operators $R_{k}(k=1,2, \ldots, d)$ should properly act on the internal space $\Omega$. In this paper, we restrict our 
considerations to the cases that all the supercharges $Q_{i}$ and $\tilde{Q}_{i}\left(\right.$ or $\left.Q_{i}^{ \pm}\right)(i=1,2, \ldots, 2\lfloor d / 2\rfloor+2)$ are well defined with the $\mathcal{N}=4\lfloor d / 2\rfloor+4$ QM SUSY algebra.

\section{REPRESENTATION OF $\mathcal{N}$-EXTENDED SUPERSYMMETRY WITH CENTRAL CHARGES}

In this section, we clarify the representation of the supersymmetry algebra derived in the previous section for the nonzero energy states.

Since the Hamiltonian $H$ and the central charges $Z_{i}$ $(i=1,2, \ldots, 2\lfloor d / 2\rfloor+2)$ commute with each other, we can introduce the simultaneous eigenstates of $H$ and $Z_{i}$. Furthermore, since the central charges satisfy the relations

$$
\left(Z_{i}\right)^{2}=H^{2} \quad(i=1,2, \ldots, 2\lfloor d / 2\rfloor+2),
$$

with

$$
H^{d-1} Z_{d+1}=H^{d-1} Z_{d+2}=Z_{1} Z_{2} \cdots Z_{d}, \quad \text { for } d=\text { even, }
$$

$$
H^{d-2} Z_{d+1}=Z_{1} Z_{2} \cdots Z_{d-1}, \quad \text { for } d=\text { odd, }
$$

the eigenvalues of $H$ and $Z_{i}$ for the nonzero energy states with $m_{n}^{2} \neq 0$ can be parametrized as follows:

$$
H \Phi_{\alpha, \vec{z}}^{(n)}(y)=m_{n}^{2} \Phi_{\alpha, \vec{z}}^{(n)}(y),
$$

$Z_{i} \Phi_{\alpha, \vec{z}}^{(n)}(y)=z_{i} m_{n}^{2} \Phi_{\alpha, \vec{z}}^{(n)}(y), \quad(i=1,2, \ldots, 2\lfloor d / 2\rfloor+2)$,

with $^{10}$

$$
\begin{gathered}
z_{i}= \pm 1 \quad(i=1,2, \ldots, 2\lfloor d / 2\rfloor+2), \\
z_{d+1}=z_{d+2}=z_{1} z_{2} \cdots z_{d} \quad \text { for } d=\text { even, } \\
z_{d+1}=z_{1} z_{2} \cdots z_{d-1} \quad \text { for } d=\text { odd }
\end{gathered}
$$

where $\vec{z}=\left(z_{1}, z_{2}, \ldots, z_{d}\right)$ and the index $\alpha$ labels the degeneracy for fixed $m_{n}$ with $\vec{z} .{ }^{11}$

It may be worthwhile explaining the physical meanings of the discrete eigenvalues $z_{i}= \pm 1$. It follows from

\footnotetext{
${ }^{10}$ It should be noted that the eigenvalues $z_{i}= \pm 1$ can be defined without ambiguity for the nonzero energy states with $m_{n}^{2} \neq 0$, and also that all of the eigenvalues $z_{i}(i=1,2, \ldots$, $2\lfloor d / 2\rfloor+2)$ are not independent but only $z_{k}(k=1,2, \ldots, d)$ are independent.

${ }^{11}$ The label $\alpha$ given in Eq. (4) corresponds to $\{\alpha, \vec{z}\}$ defined in this section.
}

expressions (17) and (18) that the central charges $Z_{i}$ essentially correspond to the reflection operators (accompanied with the Hamiltonian), so that $z_{i}$ may be interpreted as the labels for "parity" even or odd of the eigenfunctions. It should be, however, emphasized that the reflection operators $R_{k}(k=1,2, \ldots, d)$ and $P$ themselves do not commute with the supercharges $Q_{i}^{ \pm}$and hence they are not compatible with the supersymmetry. On the other hand, $Z_{i}$ commutes with all the supercharges, so that $Z_{i} / H$ (for the nonzero energy states) can be regarded as a "reflection" operator compatible with the supersymmetry.

In order to construct the representation, i.e., the supermultiplet of the supersymmetry algebra (22), (23), we first note that $Q_{i}^{-z_{i}}(i=1,2, \ldots, 2\lfloor d / 2\rfloor+2)$ acts trivially on $\Phi_{\alpha, \vec{z}}^{(n)}(y)$ with $\vec{z}=\left(z_{1}, z_{2}, \ldots, z_{d}\right)$, i.e.,

$$
Q_{i}^{-z_{i}} \Phi_{\alpha, \bar{z}}^{(n)}(y)=0 \quad(i=1,2, \ldots, 2\lfloor d / 2\rfloor+2) .
$$

This is because relation (22) implies $\left(Q_{i}^{-z_{i}}\right)^{2} \Phi_{\alpha, \vec{z}}^{(n)}(y)=0$, which leads to (32) due to the Hermitian property of the supercharges. Thus, the supercharges that act on the states $\Phi_{\alpha, \vec{z}}^{(n)}(y)$ nontrivially are given by the set of $\left\{Q_{i}^{z_{i}}(i=\right.$ $1,2, \ldots, 2\lfloor d / 2\rfloor+2)\}$ and the number of the supercharges turns out to reduce effectively to half. ${ }^{12}$

The supermultiplet associated with the state $\Phi_{\alpha, \vec{z}}^{(n)}(y)$ can be constructed in the following way. In terms of the nontrivial supercharges $Q_{i}^{z_{i}}$, it will be useful to introduce the operators

$S_{p}^{z_{2 p-1} z_{2 p}}=-i Q_{2 p-1}^{z_{2 p-1}} Q_{2 p}^{z_{2 p}} \quad(p=1,2, \ldots,\lfloor d / 2\rfloor+1)$.

They are explicitly given by

$$
\begin{aligned}
S_{p}^{z_{2 p-1} z_{2 p}}= & \left(\begin{array}{cc}
A A^{\dagger} \gamma_{(p)} \Pi_{2 p-1}^{z_{2 p-1}} \Pi_{2 p}^{z_{2 p}} & 0 \\
0 & A^{\dagger} \gamma_{(p)} \Pi_{2 p-1}^{z_{2 p-1}} \Pi_{2 p}^{z_{2 p}} A
\end{array}\right), \\
& (p=1,2, \ldots, d / 2), \\
S_{(d+2) / 2}^{z_{d+1} z_{d+2}}= & \left(\begin{array}{cc}
A A^{\dagger} \gamma^{d+1} \Pi_{d+1}^{z_{d+1}} \Pi_{d+1}^{z_{d+2}} & 0 \\
0 & -A^{\dagger} \gamma^{d+1} \Pi_{d+1}^{z_{d+1}} \Pi_{d+1}^{z_{d+2} A}
\end{array}\right),
\end{aligned}
$$

for $d=$ even and

\footnotetext{
${ }^{12}$ Note that the set of the nontrivial supercharges $\left\{Q_{i}^{z_{i}}(i=1\right.$, $2, \ldots, 2\lfloor d / 2\rfloor+2)\}$ depends on the eigenvalues $\vec{z}=\left(z_{1}, z_{2}, \ldots, z_{d}\right)$ of the state $\Phi_{\alpha, \vec{z}}^{(n)}(y)$.
} 


$$
\begin{aligned}
S_{p}^{z_{2 p-1} z_{2 p}} & =\left(\begin{array}{cc}
A A^{\dagger} \gamma_{(p)} \Pi_{d(2 p-1)}^{z_{2 p-1} \Pi_{d(2 p)}^{z_{2 p}}} & 0 \\
0 & A^{\dagger} \gamma_{(p)} \Pi_{d(2 p-1)}^{z_{2 p-1}} \Pi_{d(2 p)}^{z_{2 p}} A
\end{array}\right), \quad(p=1,2, \ldots,(d-1) / 2), \\
S_{(d+1) / 2}^{z_{d} z_{d+1}} & =\left(\begin{array}{cc}
i A A^{\dagger} \gamma^{y_{d}} \Pi_{d+1}^{z_{d}} \Pi_{d(d+1)}^{z_{d+1}} & 0 \\
0 & -i A^{\dagger} \gamma^{y_{d}} \Pi_{d+1}^{z_{d}} \Pi_{d(d+1)}^{z_{d+1}} A
\end{array}\right),
\end{aligned}
$$

for $d=$ odd, where we introduce the $p$ th internal chirality $\gamma_{(p)}=i \gamma^{y_{2 p-1}} \gamma^{y_{2 p} .}{ }^{13}$ Since $S_{p}^{z_{2 p-1} z_{2 p}}(p=1,2, \ldots,\lfloor d / 2\rfloor+1)$ commute with each other as well as $H$ and $Z_{i}(i=1$, $2, \ldots, 2\lfloor d / 2\rfloor+2)$, eigenstates of $S_{p}^{z_{2 p-1} z_{2 p}}$ can become simultaneous eigenstates of $H$ and $Z_{i}$. Furthermore, since $S_{p}^{z_{2 p-1} z_{2 p}}$ satisfy the relations $\left(S_{p}^{z_{2 p-1} z_{2 p}}\right)^{2}=H^{2}$, we can parametrize the eigenvalues of $S_{p}^{z_{2 p-1} z_{2 p}}$ as

$$
\begin{aligned}
S_{p}^{z_{2 p-1} z_{2 p}} \Phi_{s_{1} \cdots s_{p} \cdots s_{\lfloor d / 2]+1}, \vec{z}}^{(n)}(y)= & s_{p} m_{n}^{2} \Phi_{s_{1} \cdots s_{p} \cdots s_{\lfloor d / 2\rfloor+1},}^{(n)}(y) \\
& (p=1, \ldots,\lfloor d / 2\rfloor+1),
\end{aligned}
$$

with $s_{p}= \pm 1$ for the nonzero energy states. ${ }^{14}$ Here, we have replaced the index $\alpha$ by $s_{1} s_{2} \cdots s_{\lfloor d / 2\rfloor+1}$, which denote the eigenvalues of $S_{p}^{z_{2 p-1} z_{2 p}}(p=1,2, \ldots,\lfloor d / 2\rfloor+1)$.

From the relations

$$
\begin{aligned}
& Q_{i}^{z_{i}} S_{p}^{z_{2 p-1} z_{2 p}}=\left\{\begin{array}{l}
-S_{p}^{z_{2 p-1} z_{2 p}} Q_{i}^{z_{i}} \text { for } i=2 p-1,2 p, \\
+S_{p}^{z_{2 p-1} z_{2 p}} Q_{i}^{z_{i}} \text { for } i \neq 2 p-1,2 p,
\end{array}\right. \\
& {\left[Q_{i}^{z_{i}}, H\right]=\left[Q_{i}^{z_{i}}, Z_{j}\right]=0, \quad(i, j=1,2, \ldots, 2\lfloor d / 2\rfloor+2),}
\end{aligned}
$$

we find that the supercharges $Q_{2 p-1}^{z_{2 p-1}}$ (or $\left.Q_{2 p}^{z_{2 p}}\right)(p=1$, $2, \ldots,\lfloor d / 2\rfloor+1)^{15}$ flip the sign of the eigenvalues of $S_{p}^{z_{2 p-1} z_{2 p}}$ but do not change other eigenvalues. This fact implies that the set of $\left\{\Phi_{s_{1} \cdots s_{p} \cdots s_{\lfloor d / 2]+1}, \vec{z}}^{(n)}(y)\right.$ with $s_{p}=$ $\pm 1(p=1,2, \ldots,\lfloor d / 2\rfloor+1)\}$ is $2^{\lfloor d / 2\rfloor+1}$-fold degenerate and forms a supermultiplet of the $\mathcal{N}=4\lfloor d / 2\rfloor+4$ extended QM SUSY algebra with the central charges. Actually, we can explicitly construct the supermultiplet from $\Phi_{++\cdots+, \vec{z}}^{(n)}(y)$ as

\footnotetext{
${ }^{13}$ The product of $\gamma_{(p)}(p=1,2, \ldots,\lfloor d\rfloor / 2)$ equals the internal chirality $\gamma^{d+1}$ for $d=$ even and $-i \gamma^{y_{d}}$ for $d=$ odd.

${ }^{14}$ For $d=$ even, $s_{p}(p=1,2, \ldots, d / 2)$ correspond to the eigenvalues of the $p$ th internal chirality $\gamma_{(p)}$, and furthermore, $s_{(d+2) / 2}$ corresponds to the eigenvalue of $4+d$-dimensional chirality since $S_{(d+2) / 2}^{z_{d+1} z_{d+2}}$ are naively given by the product of $(-1)^{F}$ and $\gamma^{d+1}$. Thus, the eigenvalues $s_{p}(p=1,2, \ldots,(d+$ 2)/2) are independent. The similar result is also obtained for $d=$ odd.

${ }^{15}$ Since we are considering the eigenstates of $S_{p}^{z_{2 p-1} z_{2 p}}=$ $-i Q_{2 p-1}^{z_{2 p-1}} Q_{2 p}^{z_{2 p}}$, the action of $Q_{2 p}^{z_{2 p}}$ on $\Phi_{s_{1} \cdots s_{p} \cdots s_{\lfloor d / 2\rfloor+1}, z}^{(n)}(y)$ is essentially equivalent to that of $Q_{2 p-1}^{Z_{2 p-1}}$.
}

$$
\begin{aligned}
\Phi_{s_{1} s_{2} \cdots s_{\lfloor d / 2\rfloor+1}, \vec{z}}^{(n)}(y)= & \frac{1}{\left(m_{n}\right)^{s}}\left(Q_{1}^{z_{1}}\right)^{\left(1-s_{1}\right) / 2}\left(Q_{3}^{z_{3}}\right)^{\left(1-s_{2}\right) / 2} \ldots \\
& \times\left(Q_{2\lfloor d / 2\rfloor+1}^{z_{2\lfloor d / 2\rfloor+1}}\right)^{\left(1-s_{\lfloor d / 2\rfloor+1}\right) / 2} \Phi_{++\cdots+, \vec{z}}^{(n)}(y),
\end{aligned}
$$

where $s=\frac{1}{2}\left(1-s_{1}\right)+\cdots+\frac{1}{2}\left(1-s_{2\lfloor d / 2\rfloor+1}\right)$.

As we have seen so far, the number of the nontrivial supercharges reduces to half, and the $2^{\lfloor d / 2\rfloor+1}$-fold degenerate states for fixed $m_{n}$ and $\vec{z}$ are related by the reduced $2\lfloor d / 2\rfloor+2$ supercharges. This situation is known as a short representation in the context of extended supersymmetry with central charges, and the eigenstates (39) are called the 1/2-BPS states $[43,44]$.

\section{EXAMPLES}

In this section, we examine the models with the hyperrectangle and the torus extra dimensions, which realize the $\mathcal{N}=4\lfloor d / 2\rfloor+4$ extended QM SUSY, and confirm the results given in the previous section.

\section{A. Hyperrectangle}

Let us consider the example of the action (1) whose extra dimensional space $\Omega$ is given by the $d$-dimensional hyperrectangle,

$$
\Omega=\left[-\frac{L_{1}}{2}, \frac{L_{1}}{2}\right] \times \cdots \times\left[-\frac{L_{d}}{2}, \frac{L_{d}}{2}\right],
$$

where $L_{k}(k=1,2, \ldots, d)$ is the length of the $k$ th side of the hyperrectangle with the Dirichlet boundary condition imposed on the left-handed KK mode functions,

$\boldsymbol{f}_{s_{1} \cdots s_{\lfloor d / 2\rfloor}(n)}^{(n)}=0 \quad$ at $y_{k}=-\frac{L_{k}}{2}, \quad \frac{L_{k}}{2} \quad(k=1, \ldots, d)$.

This boundary condition satisfies the requirement (9), and we can confirm that all the supercharges $Q_{i}^{ \pm}$are Hermitian and well defined.

The $n$th KK mode functions $\boldsymbol{f}_{s_{1} \cdots s_{\lfloor d / 2\rfloor}}^{(n)}(y), \boldsymbol{g}_{s_{1} \cdots s_{\lfloor d / 2\rfloor}}^{(n)}(y)$ with $\mathrm{KK}$ mass $m_{n}^{2}>0$ are found to be written into the form

$$
\begin{aligned}
& \boldsymbol{f}_{s_{1} \cdots s_{\lfloor d / 2\rfloor}}^{(n)}(y)=h^{(n)}(y) \boldsymbol{e}_{s_{1} \cdots s_{\lfloor d / 2]},}, \\
& \boldsymbol{g}_{s_{1} \cdots s_{\lfloor d / 2\rfloor}}^{(n)}(y)=\frac{1}{m_{n}} A^{\dagger} h^{(n)}(y) \boldsymbol{e}_{s_{1} \cdots s_{\lfloor d / 2]}},
\end{aligned}
$$


where the second relation comes from the first equation in (7). The scalar function $h^{(n)}(y)$ and the mass eigenvalue $m_{n}$ are given by

$$
\begin{gathered}
h^{(n)}(y)=\prod_{k=1}^{d} \sqrt{\frac{2}{L_{k}}} \sin \left(\frac{n_{k} \pi}{L_{k}}\left(y_{k}+\frac{L_{k}}{2}\right)\right), \\
m_{n}^{2}=M^{2}+\sum_{k=1}^{d}\left(\frac{n_{k} \pi}{L_{k}}\right)^{2}, \quad\left(n_{k}=1,2, \ldots ; k=1, \ldots, d\right) .
\end{gathered}
$$

The $\boldsymbol{e}_{s_{1} \cdots s_{\lfloor d / 2 \mid}}$ indicate the basis vectors of the spinor space, and are chosen as eigenvectors of $\gamma_{(p)}=$ $i \gamma^{y_{2 p-1}} \gamma^{y_{2 p}}[51,52]$ :

$$
\gamma_{(p)} \boldsymbol{e}_{s_{1} \cdots s_{p} \cdots s_{\lfloor d / 2\rfloor}}=s_{p} \boldsymbol{e}_{s_{1} \cdots s_{p} \cdots s_{\lfloor d / 2]}},
$$

for all $p=1,2, \ldots,\lfloor d / 2\rfloor$, where $s_{p}= \pm 1$ represents an eigenvalue of the $p$ th internal chirality of $\gamma_{(p)}$. To fix the normalization factors of $\boldsymbol{e}_{s_{1} \cdots s_{\lfloor d / 2]}}$, we define the $\boldsymbol{e}_{s_{1} \cdots s_{\lfloor d / 2\rfloor}}$ from $\boldsymbol{e}_{+\ldots+}$ as [cf. Eqs. (20), (21), and (39)]

$$
\boldsymbol{e}_{s_{1} s_{2} \cdots s_{\lfloor d / 2\rfloor}}= \begin{cases}\left(i \gamma^{d+1} \gamma^{y_{1}}\right)^{\left(1-s_{1}\right) / 2}\left(i \gamma^{d+1} \gamma^{y_{3}}\right)^{\left(1-s_{2}\right) / 2} \cdots\left(i \gamma^{d+1} \gamma^{y_{d-1}}\right)^{\left(1-s_{d / 2}\right) / 2} \boldsymbol{e}_{++\cdots+} & (d=\text { even }), \\ \left(-\gamma^{y_{d}} \gamma^{y_{1}}\right)^{\left(1-s_{1}\right) / 2}\left(-\gamma^{y_{d}} \gamma^{y_{3}}\right)^{\left(1-s_{2}\right) / 2} \cdots\left(-\gamma^{y_{d}} \gamma^{y_{d-2}}\right)^{\left(1-s_{(d-1) / 2}\right) / 2} \boldsymbol{e}_{++\cdots+} & (d=\text { odd }) .\end{cases}
$$

Then, we can construct the eigenfunctions of the model as follows:

$$
\Phi_{s_{1} \cdots s_{\lfloor d / 2\rfloor} s_{\lfloor d / 2\rfloor+1}, \vec{z}}^{(n)}(y)= \begin{cases}\left(\begin{array}{l}
f_{s_{1} \cdots s_{\lfloor d / 2\rfloor}^{(n)}}^{(n)}(y) \\
0
\end{array}\right) & \text { for } s_{\lfloor d / 2\rfloor+1}=s_{1} s_{2} \cdots s_{\lfloor d / 2\rfloor}, \\
\left(\begin{array}{c}
0 \\
g_{s_{1} \cdots s_{\lfloor d / 2\rfloor}(n)}^{(n)}
\end{array}\right) & \text { for } s_{\lfloor d / 2\rfloor+1}=-s_{1} s_{2} \cdots s_{\lfloor d / 2\rfloor},\end{cases}
$$

where $\vec{z}=\left(z_{1}, z_{2}, \ldots, z_{d}\right)$ is given by

$$
\vec{z}= \begin{cases}\left((-)^{n_{1}+1}, \ldots,(-)^{n_{d}+1}\right) & (d=\text { even }), \\ \left((-)^{n_{1}+n_{d}}, \ldots,(-)^{n_{d-1}+n_{d}},(-)^{n_{d}+1}\right) & (d=\text { odd }) .\end{cases}
$$

Then, we can show that the eigenfunctions (47) satisfy the same relations as (39) and form the supermultiplet of the $\mathcal{N}=4\lfloor d / 2\rfloor+4$ extended QM SUSY, as the $1 / 2$-BPS states. Since the eigenvalues of $Z_{i}$ are unique at each KK level as shown in (48), the degeneracy of the 4D spectrum at each KK level is $2^{\lfloor d / 2\rfloor+1}$ and the eigenfunctions (47) are mutually related by the supercharges $Q_{i}^{z_{i}}(i=1,2, \ldots$, $2\lfloor d / 2\rfloor+2)$ at each $\mathrm{KK}$ level. It is interesting to point out that the KK mode functions $\boldsymbol{g}_{s_{1} \cdots s_{\lfloor d / 2]}}^{(n)}(y)$ are not eigenfunctions of the reflection operators $R_{k}(k=1$, $2, \ldots, d)$ and $P$, although $\boldsymbol{f}_{s_{1} \cdots s_{[d / 2]}}^{(n)}(y)$ are eigenfunctions of them. On the other hand, $\Phi_{s_{1} \cdots s_{\lfloor d / 2]} s_{\lfloor d / 2]+1}, \vec{z}}^{(n)}(y)$ are eigenfunctions of the central charges $Z_{i}(i=1,2, \ldots$, $2\lfloor d / 2\rfloor+2)$. Thus, $Z_{i}$ can be regarded as reflection operators compatible with the supersymmetry, as noticed in the previous section.

\section{B. Torus}

Next, we consider the model that the extra dimensional space $\Omega$ is given by the $d$-dimensional torus,

$$
\Omega=\left[-\frac{L_{1}}{2}, \frac{L_{1}}{2}\right) \times \cdots \times\left[-\frac{L_{d}}{2}, \frac{L_{d}}{2}\right)
$$

with the periodic boundary condition for KK mode functions,

$$
\begin{aligned}
& \boldsymbol{f}_{s_{1} \cdots s_{\lfloor d / 2]}, \vec{z}^{\prime}}^{(n)}\left(y_{1}, \ldots, y_{k}+L_{k}, \ldots y_{d}\right)=\boldsymbol{f}_{s_{1} \cdots s_{\lfloor d / 2]}, \vec{z}^{\prime}}^{(n)}\left(y_{1}, \ldots, y_{k}, \ldots, y_{d}\right), \\
& \boldsymbol{g}_{s_{1} \cdots s_{\lfloor d / 2]}, \vec{z}^{\prime}}^{(n)}\left(y_{1}, \ldots, y_{k}+L_{k}, \ldots y_{d}\right)=\boldsymbol{g}_{s_{1} \cdots s_{\lfloor d / 2]}, \vec{z}^{\prime}}^{(n)}\left(y_{1}, \ldots, y_{k}, \ldots, y_{d}\right),(k=1, \ldots, d) .
\end{aligned}
$$

The above periodic boundary condition satisfies the requirement (9), and all the supercharges are shown to be Hermitian and well defined. 
Then, the $n$th KK mode functions $\boldsymbol{f}_{s_{1} \cdots s_{[d / 2]}, \vec{z}^{\prime}}^{(n)}(y), \boldsymbol{g}_{s_{1} \cdots s_{[d / 2]}, \vec{z}^{\prime}}^{(n)}(y)$ with KK mass $m_{n}$ are found to be of the form

$$
\begin{gathered}
\boldsymbol{f}_{s_{1} \cdots s_{\lfloor d / 2]}, \vec{z}^{\prime}}^{(n)}(y)=h_{\vec{z}^{\prime}}^{(n)}(y) \boldsymbol{e}_{s_{1} \cdots s_{\lfloor d / 2]},}, \quad \boldsymbol{g}_{s_{1} \cdots s_{\lfloor d / 2]}, \vec{z}^{\prime}}^{(n)}(y)=\frac{1}{m_{n}} A^{\dagger} h_{\vec{z}^{\prime}}^{(n)}(y) \boldsymbol{e}_{s_{1} \cdots s_{\lfloor d / 2]},}, \\
h_{\vec{z}^{\prime}}^{(n)}(y)=\prod_{k=1}^{d} h_{z_{k}^{\prime}}^{\left(n_{k}\right)}(y), \quad h_{z_{k}^{\prime}}^{\left(n_{k}\right)}(y)= \begin{cases}\sqrt{\frac{2}{L_{k}}} \cos \left(\frac{2 n_{k} \pi}{L_{k}} y_{k}\right) & \left(z_{k}^{\prime}=+1\right), \\
\sqrt{\frac{2}{L_{k}}} \sin \left(\frac{2 n_{k} \pi}{L_{k}} y_{k}\right) & \left(z_{k}^{\prime}=-1\right),\end{cases} \\
m_{n}^{2}=M^{2}+\sum_{k=1}^{d}\left(\frac{2 n_{k} \pi}{L_{k}}\right)^{2}, \quad\left(n_{k}=0,1,2, \ldots ; k=1, \ldots, d\right),
\end{gathered}
$$

where $z_{k}^{\prime}= \pm 1$ and $\vec{z}^{\prime}=\left(z_{1}^{\prime}, \ldots, z_{d}^{\prime}\right)$.

Then, we can construct the eigenfunctions of the model as

$$
\Phi_{s_{1} \cdots s_{\lfloor d / 2\rfloor} s_{\lfloor d / 2\rfloor+1}, \vec{z}}^{(n)}(y)=\left\{\begin{array}{cc}
\left(\begin{array}{c}
f_{s_{1} \cdots s_{\lfloor d / 2\rfloor}, \vec{z}^{\prime}}^{(n)}(y) \\
0
\end{array}\right) & \text { for } s_{\lfloor d / 2\rfloor+1}=s_{1} s_{2} \cdots s_{\lfloor d / 2\rfloor}, \\
\left(\begin{array}{c}
0 \\
g_{s_{1} \cdots s_{\lfloor d / 2\rfloor}, \vec{z}^{\prime}}^{(n)}
\end{array}\right) & \text { for } s_{\lfloor d / 2\rfloor+1}=-s_{1} s_{2} \cdots s_{\lfloor d / 2\rfloor},
\end{array}\right.
$$

where $\vec{z}$ is given by

$$
\vec{z}= \begin{cases}\left(z_{1}^{\prime}, \ldots, z_{d}^{\prime}\right) & (d=\text { even }), \\ \left(z_{d}^{\prime} z_{1}^{\prime}, \ldots, z_{d}^{\prime} z_{d-1}^{\prime}, z_{d}^{\prime}\right) & (d=\text { odd }) .\end{cases}
$$

Then, we can show that the eigenfunctions (54) satisfy the same relation as (39) and form the supermultiplet of the $\mathcal{N}=4\lfloor d / 2\rfloor+4$ extended QM SUSY, as the 1/2-BPS states. However, unlike the hyperrectangle case, both of the eigenstates with $z_{k}=+1$ and -1 for $n_{k} \neq 0 \quad(k=1$, $2, \ldots, d)$ are degenerate in the $4 \mathrm{D}$ mass spectrum. This implies that the additional degeneracy $2^{d-N_{0}}$ appears in the $4 \mathrm{D}$ spectrum, where $N_{0}$ is the number of zeros in $\left\{n_{1}, n_{2}, \ldots, n_{d}\right\} .{ }^{16}$ The origin of the degeneracy comes from the extra degrees of freedom with respect to the parity even or odd for each reflection: $y_{k} \rightarrow-y_{k}$ in $h_{\bar{z}^{\prime}}^{(n)}(y)$. Therefore, the $4 \mathrm{D}$ mass spectrum is $\left(2^{2\lfloor d / 2\rfloor+1} \times 2^{d-N_{0}}\right)$-fold degenerate for the KK modes labeled by $\left\{n_{1}, n_{2}, \ldots, n_{d}\right\}$.

\section{SUMMARY AND DISCUSSION}

In this paper, we have revealed that the $\mathcal{N}$-extended QM SUSY with the central charges is hidden in the 4D mass spectrum of the higher dimensional Dirac action. The supercharges are obtained as the extension of the $\mathcal{N}=2$ QM SUSY based on the algebraic properties of the internal gamma matrices and the reflection symmetries of the extra

\footnotetext{
${ }^{16}$ Note that when $n_{k}=0, h_{z_{k}^{\prime}}^{\left(n_{k}\right)}(y)$ for $z_{k}^{\prime}=-1$ is trivial, i.e., $h_{z_{k}^{\prime}}^{(0)}(y)=0$. Thus, there is no degeneracy in $h_{z_{k}^{\prime}}^{\left(n_{k}\right)}(y)$ for $n_{k}=0$.
}

dimensions. The central charges are interpreted as the supersymmetric extension of the reflection operators.

We have also examined the representation of the extended supersymmetry algebra and found that the supermultiplet corresponds to the short multiplet of the 1/2-BPS states. Furthermore, we have explicitly confirmed that the KK mode functions in the models of the hyperrectangle and the torus extra dimensions can be properly classified by the representations of the $\mathcal{N}=4\lfloor d / 2\rfloor+4$ extended QM SUSY algebra with the central charges.

In this paper, we have restricted to the cases that all the $4\lfloor d / 2\rfloor+4$ supercharges are well defined. Other boundary conditions, other extra dimensions and nontrivial background fields would break (or partially break) the extended supersymmetry. For example, if there are no reflection symmetries in extra dimensions, the geometric supercharges (13) and (14) [and also the supercharges (19)] become ill defined, although the algebraic supercharges can be well defined in this case with suitable boundary conditions. Therefore, it would be of great importance to clarify how the extended supersymmetry found in this paper is broken by the choice of boundary conditions, extra dimensional spaces, and background fields.

It is interesting to note that there are possibilities that further structures might be hidden in the 4D mass spectrum in general settings. The central charges in our models result from the symmetries of the extra dimensions. Thus, we can expect that new types of central charges will appear in models with other symmetries. Furthermore, since it is known that central charges are closely related to topological properties $[43,53,54]$, it is also interesting to investigate models of curved 
extra dimensions or background fields with nontrivial topologies, e.g., sphere, soliton, magnetic flux, etc.

In addition, since we have obtained the new extended supersymmetry with the central charges, it would be worthwhile to search for new types of exactly solvable models by use of this supersymmetry. The issues mentioned above remain to be done in future works.

\section{ACKNOWLEDGMENTS}

This work is supported in part by Grants-in-Aid for Scientific Research [No. 18K03649 (Y. F. and M. S.)] from the Ministry of Education, Culture, Sports, Science and Technology (MEXT) in Japan. K. N. had been funded by the research grant (No. PG052102) from Korea Institute for Advanced Study (KIAS), and has been supported by the European Union through the European Regional Development Fund - the Competitiveness and Cohesion Operational Programme (KK.01.1.1.06), the European Union's Horizon 2020 research and innovation program under the Twinning Grant Agreement No. 692194, RBI-TWINNING, and the grant funded from the European Structural and Investment Funds, No. RBI-TWINN-SIN.
[1] E. Witten, Dynamical breaking of supersymmetry, Nucl. Phys. B188, 513 (1981).

[2] F. Cooper, A. Khare, and U. Sukhatme, Supersymmetry and quantum mechanics, Phys. Rep. 251, 267 (1995).

[3] C. Quesne and V. M. Tkachuk, Dirac oscillator with nonzero minimal uncertainty in position, J. Phys. A 38, 1747 (2005).

[4] B. Bagchi, C. Quesne, and R. Roychoudhury, Isospectrality of conventional and new extended potentials, second-order supersymmetry and role of PT symmetry, Pramana 73, 337 (2009).

[5] S. Odake and R. Sasaki, Infinitely many shape invariant potentials and new orthogonal polynomials, Phys. Lett. B 679, 414 (2009).

[6] David J. Fernández C, Supersymmetric quantum mechanics, AIP Conf. Proc. 1287, 3 (2010).

[7] C. Pedder, J. Sonner, and D. Tong, The geometric phase in supersymmetric quantum mechanics, Phys. Rev. D 77, 025009 (2008).

[8] S. Ohya, Non-Abelian monopole in the parameter space of point-like interactions, Ann. Phys. (Amsterdam) 351, 900 (2014).

[9] S. Ohya, BPS monopole in the space of boundary conditions, J. Phys. A 48, 505401 (2015).

[10] P. Claus, M. Derix, R. Kallosh, J. Kumar, P. K. Townsend, and A. Van Proeyen, Black Holes and Superconformal Mechanics, Phys. Rev. Lett. 81, 4553 (1998).

[11] G. W. Gibbons and P. K. Townsend, Black holes and Calogero models, Phys. Lett. B 454, 187 (1999).

[12] R. Britto-Pacumio, J. Michelson, A. Strominger, and A. Volovich, Lectures on superconformal quantum mechanics and multi-black hole Moduli spaces, NATO Sci. Ser. C 556, 255 (2000).

[13] S. Bellucci, A. Galajinsky, E. Ivanov, and S. Krivonos, $\operatorname{AdS}(2) / C F T(1)$, canonical transformations and superconformal mechanics, Phys. Lett. B 555, 99 (2003).

[14] I. Bakas, Energy-momentum/Cotton tensor duality for AdS(4) black holes, J. High Energy Phys. 01 (2009) 003.

[15] W. Fu, D. Gaiotto, J. Maldacena, and S. Sachdev, Supersymmetric Sachdev-Ye-Kitaev models, Phys. Rev. D 95, 026009 (2017).
[16] T. Li, J. Liu, Y. Xin, and Y. Zhou, Supersymmetric SYK model and random matrix theory, J. High Energy Phys. 06 (2017) 111.

[17] J. Yoon, Supersymmetric SYK model: Bi-local collective Superfield/Supermatrix formulation, J. High Energy Phys. 10 (2017) 172.

[18] J. Murugan, D. Stanford, and E. Witten, More on supersymmetric and $2 d$ analogs of the SYK model, J. High Energy Phys. 08 (2017) 146.

[19] N. Hunter-Jones and J. Liu, Chaos and random matrices in supersymmetric SYK, J. High Energy Phys. 05 (2018) 202.

[20] C. S. Lim, T. Nagasawa, M. Sakamoto, and H. Sonoda, Supersymmetry in gauge theories with extra dimensions, Phys. Rev. D 72, 064006 (2005).

[21] C. S. Lim, T. Nagasawa, S. Ohya, K. Sakamoto, and M. Sakamoto, Supersymmetry in 5D gravity, Phys. Rev. D 77, 045020 (2008).

[22] C. S. Lim, T. Nagasawa, S. Ohya, K. Sakamoto, and M. Sakamoto, Gauge fixing and residual symmetries in gauge/ gravity theories with extra dimensions, Phys. Rev. D 77, 065009 (2008).

[23] T. Nagasawa, S. Ohya, K. Sakamoto, and M. Sakamoto, Emergent supersymmetry in warped backgrounds, symmetry, integrability and geometry: Methods and applications, SIGMA 7, 065 (2011).

[24] Y. Fujimoto, K. Hasegawa, K. Nishiwaki, M. Sakamoto, and K. Tatsumi, 6d Dirac fermion on a rectangle; scrutinizing boundary conditions, mode functions and spectrum, Nucl. Phys. B922, 186 (2017).

[25] Y. Fujimoto, K. Hasegawa, K. Nishiwaki, M. Sakamoto, and K. Tatsumi, Supersymmetry in the 6D Dirac action, Prog. Theor. Exp. Phys. (2017), 073B03.

[26] David J. Fernández C, Trends in supersymmetric quantum mechanics, arXiv:1811.06449.

[27] A. Pashnev and F. Toppan, On the classification of $\mathrm{N}$-extended supersymmetric quantum mechanical systems, J. Math. Phys. (N.Y.) 42, 5257 (2001).

[28] Z. Kuznetsova, M. Rojas, and F. Toppan, Classification of irreps and invariants of the $N$-extended supersymmetric quantum mechanics, J. High Energy Phys. 03 (2006) 098. 
[29] M. Faux and S. J. Gates, Jr., Adinkras: A graphical technology for supersymmetric representation theory, Phys. Rev. D 71, 065002 (2005).

[30] M. de Crombrugghe and V. Rittenberg, Supersymmetric quantum mechanics, Ann. Phys. (N.Y.) 151, 99 (1983).

[31] P. Howe, S. Penati, M. Pernici, and P. Townsend, Wave equations for arbitrary spin from quantization of the extended supersymmetric spinning particle, Phys. Lett. B 215, 555 (1988).

[32] V. Akulov and M. Kudinov, Extended supersymmetric quantum mechanics, Phys. Lett. B 460, 365 (1999).

[33] T. Nagasawa, M. Sakamoto, and K. Takenaga, Supersymmetry and discrete transformations on $S 1$ with point singularities, Phys. Lett. B 583, 357 (2004).

[34] T. Nagasawa, M. Sakamoto, and K. Takenaga, Extended supersymmetry and its reduction on a circle with point singularities, J. Phys. A 38, 8053 (2005).

[35] E. A. Ivanov, S. O. Krivonos, and A. I. Pashnev, Partial supersymmetry breaking in $N=4$ supersymmetric quantum mechanics, Classical Quantum Gravity 8, 19 (1991).

[36] J. Niederle and A. G. Nikitin, Extended SUSY with central charges in quantum mechanics, Proc. Inst. Math. NAS (Ukraine) 43, 497 (2002).

[37] M. Faux and D. Spector, Duality and central charges in supersymmetric quantum mechanics, Phys. Rev. D 70, 085014 (2004).

[38] S. Bellucci, S. Krivonos, and A. Shcherbakov, Twodimensional $N=8$ supersymmetric mechanics in superspace, Phys. Lett. B 612, 283 (2005).

[39] P. Fayet, Fermi-Bose hypersymmetry, Nucl. Phys. B113, 135 (1976).

[40] P. Fayet, Spontaneous generation of massive multiplets and central charges in extended supersymmetric theories, Nucl. Phys. B149, 137 (1979).

[41] P. Fayet, $N=2$ Extended supersymmetric GUTs: Gauge boson/Higgs boson unification, mass spectrum and central charges, Nucl. Phys. B246, 89 (1984).
[42] P. Fayet, Six-dimensional supersymmetric QED, $R$ invariance and $N=2$ supersymmetry breaking by dimensional reduction, Nucl. Phys. B263, 649 (1986).

[43] E. Witten and D. I. Olive, Supersymmetry algebras that include topological charges, Phys. Lett. 78B, 97 (1978).

[44] J. Wess and J. Bagger, Supersymmetry and supergravity, 2nd ed. (Princeton University Press, Princeton, NJ, 1992).

[45] M. K. Prasad and C. M. Sommerfield, An Exact Classical Solution for the 't Hooft Monopole and the Julia-Zee Dyon, Phys. Rev. Lett. 35, 760 (1975).

[46] E. B. Bogomolny, Stability of classical solutions, Yad. Fiz. 24, 861 (1976) [Sov. J. Nucl. Phys. 24, 449 (1976)].

[47] Y. Fujimoto, K. Hasegawa, K. Nishiwaki, M. Sakamoto, K. Tatsumi, and I. Ueba, Extended supersymmetry in Dirac action with extra dimensions, J. Phys. A 51, 435201 (2018).

[48] R. Brauer and H. Weyl, Spinors in $n$ dimensions, Am. J. Math. 57, 425 (1935).

[49] C. Wetterich, Massless spinors in more than fourdimensions, Nucl. Phys. B211, 177 (1983).

[50] T. Kugo and P. K. Townsend, Supersymmetry and the division algebras, Nucl. Phys. B221, 357 (1983).

[51] J. Polchinski, String Theory: Volume 1, An Introduction to the Bosonic String (Cambridge University Press, Cambridge, England, 1998).

[52] J. Polchinski, String Theory: Volume 2, Superstring Theory and Beyond (Cambridge University Press, Cambridge, England, 1998).

[53] N. Seiberg and E. Witten, Electric-magnetic duality, monopole condensation, and confinement in $N=2$ supersymmetric Yang-Mills theory, Nucl. Phys. B426, 19 (1994); B430, 485 (1994).

[54] N. Seiberg and E. Witten, Monopoles, duality and chiral symmetry breaking in $N=2$ supersymmetric QCD, Nucl. Phys. B431, 484 (1994). 